\title{
Phylogeny, Regression, and the Allometry of Physiological Traits
}

Article in The American Naturalist · October 2007

DOI: $10.1086 / 519459 \cdot$ Source: PubMed

CITATIONS

28

7 authors, including:

\section{Stanley J. Kemp}

University of Baltimore

13 PUBLICATIONS 358 CITATIONS

SEE PROFILE

\section{James N. McNair}

Grand Valley State University 40 PUBLICATIONS 1,187 CITATIONS

SEE PROFILE
READS

46

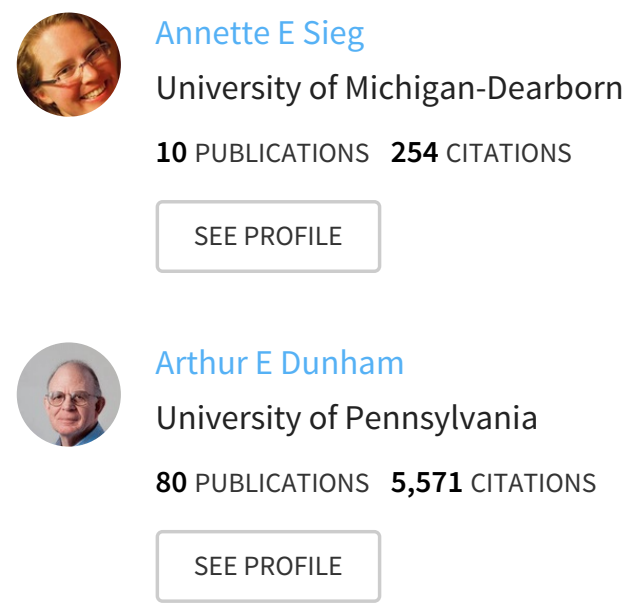

Some of the authors of this publication are also working on these related projects:

Project Temperature dependent changes in mitochondrial efficiency View project

The Stockholm Paradigm: testing the elements of a paradigm in evolution using theoretical and empirical data View project 


\title{
Phylogeny, Regression, and the Allometry of Physiological Traits
}

\author{
Michael P. O'Connor, ${ }^{1, *}$ Salvatore J. Agosta, ${ }^{2, \dagger}$ Frank Hansen, ${ }^{2, \star}$ Stanley J. Kemp, ${ }^{2, \S}$ Annette E. Sieg, ${ }^{1, \|}$ \\ James N. McNair, ${ }^{3, *}$ and Arthur E. Dunham ${ }^{2, * *}$
}

1. Department of Bioscience and Biotechnology, Drexel University, Philadelphia, Pennsylvania 19104;

2. Department of Biology, University of Pennsylvania, Philadelphia, Pennsylvania 19104;

3. Patrick Center for Environmental Research, Academy of Natural Sciences of Philadelphia, Philadelphia, Pennsylvania 19103

Submitted July 12, 2006; Accepted February 27, 2007;

Electronically published July 24, 2007

Online enhancement: appendix.

ABStRACt: Physiological and ecological allometries often pose linear regression problems characterized by (1) noncausal, phylogenetically autocorrelated independent $(x)$ and dependent $(y)$ variables (characters); (2) random variation in both variables; and (3) a focus on regression slopes (allometric exponents). Remedies for the phylogenetic autocorrelation of species values (phylogenetically independent contrasts) and variance structure of the data (reduced major axis [RMA] regression) have been developed, but most functional allometries are reported as ordinary least squares (OLS) regression without use of phylogenetically independent contrasts. We simulated Brownian diffusive evolution of functionally related characters and examined the importance of regression methodologies and phylogenetic contrasts in estimating regression slopes for phylogenetically constrained data. Simulations showed that both OLS and RMA regressions exhibit serious bias in estimated regression slopes under different circumstances but that a modified orthogonal (least squares variance-oriented residual [LSVOR]) regression was less biased than

* Corresponding author; e-mail: mike.oconnor@drexel.edu.

† E-mail: agosta@sas.upenn.edu.

₹ E-mail: fhansen@sas.upenn.edu.

$\$$ Present address: Department of Biology, Pennsylvania State University, Berks Campus, Reading, Pennsylvania 19607; e-mail: skemp@sas.upenn.edu.

" E-mail: aes48@drexel.edu.

\# E-mail: mcnair@acnatsci.org.

** E-mail: adunham@sas.upenn.edu.

Am. Nat. 2007. Vol. 170, pp. 431-442. () 2007 by The University of Chicago. 0003-0147/2007/17003-41955\$15.00. All rights reserved.

DOI: $10.1086 / 519459$ either OLS or RMA regressions. For strongly phylogenetically structured data, failure to use phylogenetic contrasts as regression data resulted in overestimation of the strength of the regression relationship and a significant increase in the variance of the slope estimate. Censoring of data sets by simulated extinction of taxa did not affect the importance of appropriate regression models or the use of phylogenetic contrasts.

Keywords: allometry, body size, phylogeny, regression.

Frequently, in comparative studies, the relationship between the factors of interest is conflated with phylogenetic influences, complicating both statistical analyses and inferences about the factors (Felsenstein 1985; Freckleton et al. 2002). When we are interested in quantifying the relations among physiological and ecological variables (e.g., the relation between body size and longevity), the problems include (1) lack of phylogenetic independence of the variables (e.g., body size and longevity) for two related species, which violates the assumptions of most regression or correlation analyses; (2) consequently inflated estimates of degrees of freedom, which complicates tests of significance; (3) the possibility that differentiation among higher-order taxa and subsequent phylogenetic canalization might alter the apparent relation between the two variables, even creating apparent functional relationships where none exist (Felsenstein 1985); and (4) the possibility that neither of the variables is an independent variable; that is, each of the variables actually depends on a third variable not available for analysis (Legendre and Legendre 1998).

Felsenstein (1985) used contrasts among species (and among hypothetical, reconstructed ancestors) to minimize phylogenetic influences in attempts to construct such functional relationships and remedy problems $1-3$ listed above. With subsequent modifications, his approach has become a standard methodology for comparative studies (Freckleton et al. 2002).

As an example of the importance of such regressions on functional data, we focus on allometric effects on metabolic rate, longevity, population density, life history, and 
evolution, which have recently been reemphasized as part of the metabolic theory of ecology (West et al. 1997; Brown et al. 2004; Gillooly et al. 2005; West and Brown 2005). Part of the debate about such allometric effects has centered on the precise slope of the relationship between whole animal metabolic rate and body mass (more specifically, their logarithms) in a variety of groups including bacteria, plants, and animals, with particular emphasis on vertebrates and especially mammals. Recent treatments (e.g., Peters 1983; Calder 1984; Schmidt-Nielsen 1984) adopted a slope of 0.75 for this regression, and Brown et al. (2004) and Savage et al. (2004) adopt that as part of their synthesis. Others argue that a slope of 0.67 is more consistent with the data (Dodds et al. 2001; White and Seymour 2003, 2005), that the slope varies with the level of metabolic exertion of an animal (White and Seymour 2005), or that the slope varies among species, clades, or sizes of mammals (Dodds et al. 2001; Glazier 2005). Others note that slope estimates are suspect because rarely do such analyses use phylogenetic contrasts to estimate the required slope (LaBarbera 1989; Martin et al. 2005). Symonds and Elgar (2002) found sufficient phylogenetic signal in mammalian metabolic data that different phylogenies gave different estimates of allometric exponents. LaBarbera (1989) argued that most allometric analyses of metabolic rates underestimated the slope of the relationship because they used model 1 regressions (ordinary least squares [OLS] regressions, appropriate for regressions when the error in the "independent variable" is small) rather than a model 2 regression (appropriate when the independent variable is a random variable with measurable error; Sokal and Rohlf 1995). Indeed, when performed on the same data, model 1 regressions yield lower slopes than model 2 regressions (LaBarbera 1989; Legendre and Legendre 1998), although the higher slopes of model 2 regressions may constitute an overcorrection (Carroll and Ruppert 1996). Finally, most software that performs phylogenetic contrasts typically uses model 1 rather than model 2 regressions and depends critically on the use of hypothetical ancestral species, and it may, therefore, be suspect (Isler et al. 2002; Martin et al. 2005). Under these circumstances, we argue that disputes about the precise values of slopes without first determining which analytic methods are appropriate are premature.

Researchers often debate the advisability of using model 1 or model 2 regression and phylogenetic contrasts on the basis of theoretical considerations (e.g., Glazier 2005). To investigate the possibility that regression and phylogenetic techniques might (possibly synergistically) misestimate metabolic and other physiological allometries, we simulated evolutionary differentiation in a clade and estimated the slope of the regression of two variables by a series of techniques. We sought to answer three questions: (1) To what extent do different regression models affect estimated slopes when applied to a phylogenetically structured regression problem? (2) To what extent does the use of phylogenetic contrasts as suggested by Felsenstein (1985) affect the regression of two variables with an underlying functional relationship using each of the regression models (model 1, model 2, others)? (3) To what extent do different methods of constructing simple phylogenetic contrasts and reconstructing ancestral species affect the estimated slopes of the functional relationships using different regression methods? In each case, we estimated slopes from the simulated data using various regression techniques, with and without phylogenetic contrasts, and compared the estimated slopes with those expected as the outcome of the simulation.

\section{Methods \\ Simulating Evolution}

We simulated evolution within a clade as a discrete-time Brownian diffusion process with each taxon's characters iteratively and randomly diffusing through the potential character space and each taxon having a fixed probability of fissioning into two daughter taxa at each time step (fig. 1). While other models of evolution are available and important (e.g., Hansen 1997), Brownian diffusion is a simple null model and serves as the basis for much of the theory underlying phylogenetic analyses (Felsenstein 1985; Rohlf 2001; Freckleton et al. 2002).

At each time step, the algorithm iterated through all extant taxa, allowing characters to evolve and taxa to bifurcate. We modeled the evolution of correlated characters by relating each character to a single driving variable that was assumed not to be available for analysis. Thus, character diffusion for each taxon at each time step depended on a hidden normally distributed master variable $(\Delta T)$ with a mean of 0 and variance (constant over all extant taxa) of $\sigma_{T}^{2}$. Changes in each of the character states for the taxon depended on $\Delta T$ but had an additional random "error" component as in equation (1):

$$
\Delta x_{i, t}=S_{x} \Delta T_{i, t}+\sigma_{x} \varepsilon_{i, t, x}
$$

where $\Delta x_{i, t}$ is the change in character $x$ for taxon $i$ at time $t, \Delta T_{i, t}$ is the value of $\Delta T$ for taxon $i$ at time $t$, and $\varepsilon_{i, t, x}$ is the random error for character $x$ in taxon $i$ at time $t$ (normally distributed with mean $=0$ and variance $=1$ ). The symbol $S_{x}$ is a multiplier giving the functional dependence of the character $x$ on the master variable $\Delta T$ that remained constant for all taxa throughout the simulation. The magnitude of the variation in $x$ that was 


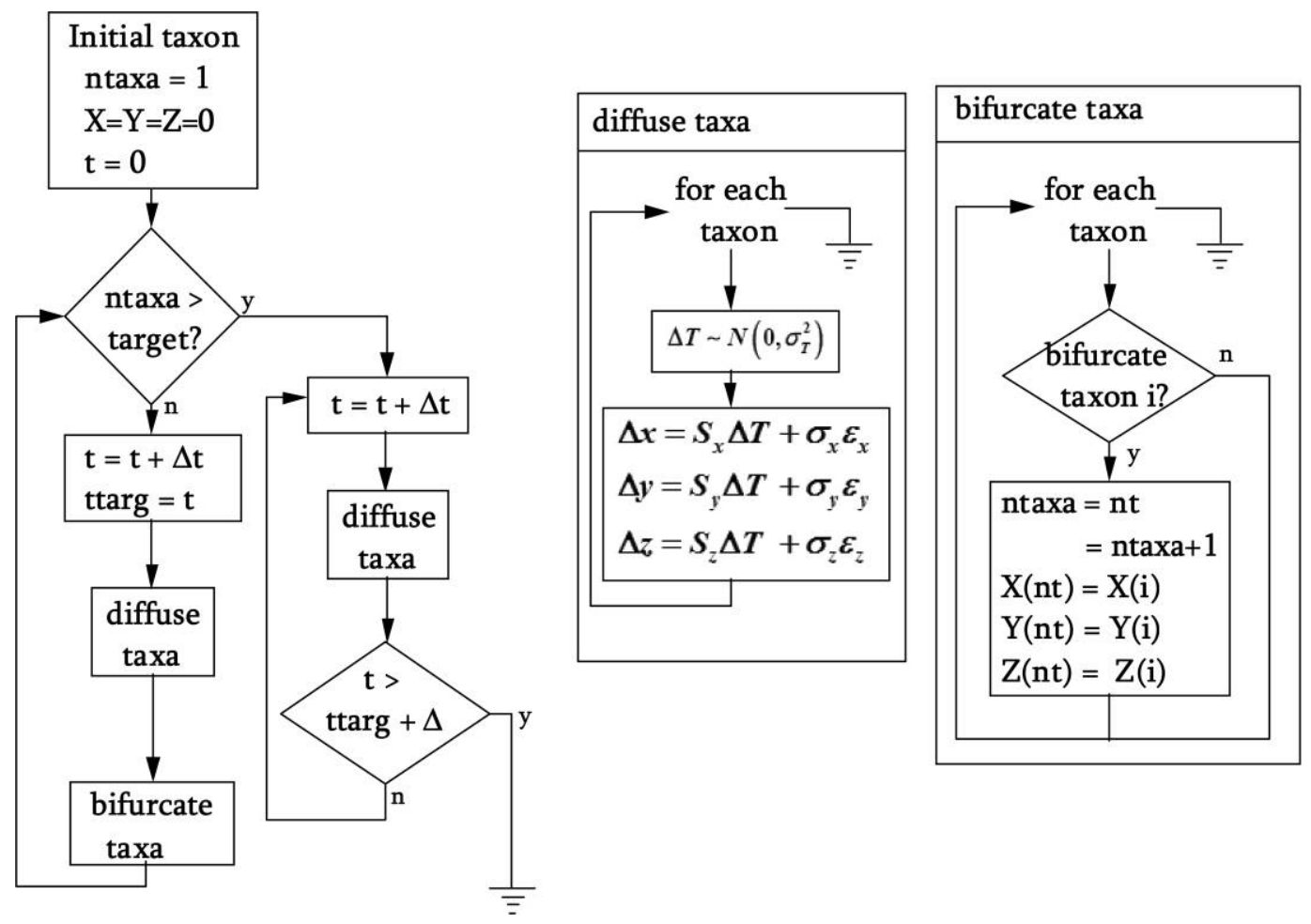

Figure 1: Flow diagram for simulations of Brownian character diffusion. Each step involved both character diffusion for each taxon and a low probability of bifurcation for each taxon.

uncorrelated with $\Delta T$ was controlled by $\sigma_{x}$, which was related to $S_{x}$ as in equation (2):

$$
\sigma_{x}=E \times \sigma_{T} \times S_{x},
$$

where $\sigma_{T}$ was the standard deviation of $\Delta T$ and $E$ was an error multiplier that typically ranged from 0.01 to 0.5 and was an important simulation parameter.

We simulated the evolution of three characters- $x, y$, and $z$-each with its own dependence on $\Delta T$. Results for each of the two-variable comparisons were similar, so we present only results for $x$ and $y$ with $S_{x}=4$ and $S_{y}=3$. Regressions of $y$ on $x$ with those values of $S_{x}$ and $S_{y}$ have an expected slope of 0.75 .

Each taxon had a fixed probability of bifurcating $\left(P_{\mathrm{b}}\right)$ into two daughter taxa at each time step (fig. 1). Those daughter taxa subsequently evolved independently for the rest of the simulation. Hence, within the simulations, there was no tendency for related taxa to remain alike because they continued to share common habitats or habits after bifurcating; that is, the only reason for similarity among taxa is shared ancestry. We used several probabilities for taxon bifurcation, but as long as that probability was low $(<3 \%)$, results were independent of the bifurcation prob- ability, and $P_{\mathrm{b}}$ was 0.01 for all simulations presented here. Because simulations started with a single root taxon (with $x=y=z=0$ ) and the probability of bifurcation was low, simulations sometimes ran for as many as $200-300$ time steps before the first bifurcation occurred, which allowed the root of the phylogeny to undergo substantial character diffusion before taxon divergence began.

Simulations ran until a target number of taxa (20-300) were present (fig. 1). Once the target number of taxa was reached, the simulation continued for a further $\Delta$ time steps (fig. $1 ; \Delta=30$ in all simulations presented here) with taxa diffusing but not bifurcating to allow the newest taxa to diverge from their sister taxa.

A typical tree structure for a simulation with 50 terminal taxa is shown in figure 2. The taxonomic order does not correspond to any of the character states. Its major significance is that all of the terminal taxa in a given clade will have contiguous integer values. Taxonomic order values for ancestor taxa are the means of the values of two descendant taxa. Note that some clades are highly species rich while others are not (e.g., taxa with taxonomic orders 28,29 , and 50 in fig. 2). Thus, some terminal taxa will be compared with distantly ancestral taxa in doing phylogenetic contrasts. 


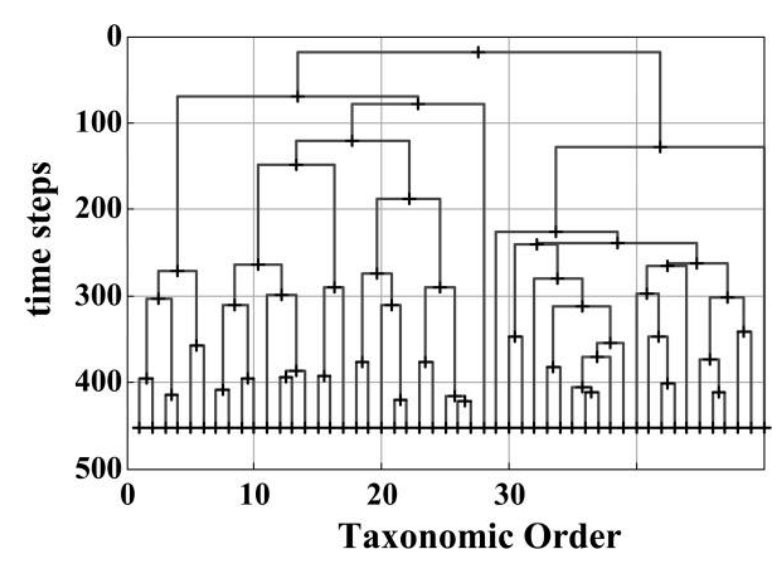

Figure 2: Typical tree generated by the diffusion algorithm for a simulation with 50 terminal taxa. Horizontal lines represent times at which a taxon bifurcated into two daughter taxa. Taxonomic order is an arbitrary variable useful for ordering taxa but unrelated to character values.

Simulations were run in two modes. We simulated trees in which all parameters $\left(P_{\mathrm{b}}, \sigma_{T}, S_{x}, S_{y}, \sigma_{x}, \sigma_{y}\right)$ were constant throughout the simulation (constant evolution or diversification). Because rates of divergence by radiation vary through time (and many models contemplate trees with rapid diversification near the root of the tree), we ran a second set of simulations in which $\sigma_{T}$ was large initially but decreased stepwise by a factor of 10 when the number of taxa reached the square root of the target number of taxa (rapid early diversification). This mode of simulation increased character differences among clades and created a stronger phylogenetic signal in the character regressions. We measured phylogenetic signal strength by comparing character variances within and among clades in simulated phylogenies. We simulated trees with 100 terminal taxa and designated the first 10 taxa generated as roots of the clades. We calculated the ratio of the summed squared character deviations among clades to the within-clade sum squares $\left(\mathrm{SS}_{\mathrm{b} / \mathrm{w}} / \mathrm{SS}_{\mathrm{w} / \mathrm{in}}\right)$. For trees with constant diversification rates, these ratios averaged 1.3-1.4 regardless of the error multiplier $(E)$ used. For trees with rapid early diversification, $\mathrm{SS}_{\mathrm{b} / \mathrm{w}} / \mathrm{SS}_{\mathrm{w} / \mathrm{in}}$ ranged from 4 to 70 depending on the error multiplier used.

Most of our simulations did not include taxon extinction; that is, all taxa either bifurcated into descendant taxa or persisted until the end of the simulation. To determine whether extinction might alter the simulation results, we simulated trees with 300 terminal taxa and then progressively pruned the trees by randomly selecting terminal and ancestral taxa for deletion. When a taxon was removed, all of its descendant taxa were also removed. After each deletion, results for the remaining tree were compared with those for the original fully populated tree. Iterative pruning continued until the number of remaining taxa reached 30 .

\section{Regression Models}

Model 1 regression is the well-known ordinary least squares (OLS) regression (Sokal and Rohlf 1995). The independent variable is assumed to have negligible error variance for each data point. Slope estimates are derived from the normal equations that result from minimizing the summed squares of the $y$ residuals (deviations of the actual dependent variable value from that predicted by a straight line at a fixed $x$ value). One can also regress the independent, or $x$, variable on the dependent $(y)$ variable, a process we designate inverse least squares (ILS) regression.

Model 2 regression, on the other hand, assumes that both variables are random variables with some random component in both $x$ and $y$ directions. The labels "dependent" and "independent" may or may not be meaningful. Both variables may, as happens here, depend on a third unmeasured variable (Sokal and Rohlf 1995; Legendre and Legendre 1998). The regression minimizes the magnitude of distances of each point perpendicular (normal) to the regression line. That distance typically includes both $x$ and $y$ components.

Several forms of model 2 regression are available. Reduced major axis (RMA) regression computes the regression line using standardized variables as in equation (3):

$$
x^{\prime}=\frac{x-\bar{x}}{S_{x}}
$$

The computed slope is that of the first principal component, or the dominant eigenvector, of the correlation matrix. The slope of $y$ on $x$ in terms of the original variables can be obtained by multiplying by $\sigma_{x} / \sigma_{y}$. It can also be calculated as the geometric mean of the $y$ on $x$ slopes calculated by OLS and ILS regressions. Major axis (MA) regression performs a similar regression on the native (unstandardized) $x$ and $y$ values. Finally, a ranged major axis regression can be calculated if each of the variables is divided by its range (Legendre and Legendre 1998).

When variables are tightly correlated $\left(r^{2}\right.$ close to 1.0$)$, slopes for all regression models and methods are very similar. At lower $r^{2}$ values, RMA $y$-on- $x$ slopes are noticeably steeper than OLS slopes and shallower than ILS $y$-on- $x$ slopes calculated from the same data. Researchers differ on when to use model 1 or variants of model 2 regression but commonly suggest using model 1 regression when the variance of $x$ for any given $x, y$ pair is much smaller than the $y$ variance or when the purpose of the regression is to estimate a $y$ value given a particular $x$ value (within the 
measured range of $x^{\prime}$ s). Alternatively, when the residual $x$ variance is appreciable and the slope of the regression is the objective of the regression, most suggest using RMA regression (summary in Quinn and Keough 2002). In an extensive review, Warton et al. (2006) recommended model 2 regression for regressions using phylogenetic contrasts. Here, we focus on the relative utility of different regression models in estimating slopes for physiological relationships.

An important limitation of MA or RMA regressions is that they assume that the variances (random or due to measurement errors) for both $x$ and $y$ variables are equal. For allometric and other comparative regressions, we often have available not only the mean values for a trait (e.g., of mass and metabolism) for each species but also a measure of the trait variation (due either to measurement error or to natural variation in the trait; e.g., not all members of a species have the same mass). "Orthogonal" regressions (Jolicouer and Heusner 1971; Jolicouer 1975; Carroll and Ruppert 1996) weight the importance of the $x$ and $y$ errors by the magnitude of those errors. We developed an orthogonal regressor that calculates residuals along a fixed slope and minimizes the sum of those squared residuals. We computed residuals along a slope ( $\left(\operatorname{Dir}_{\text {res }}\right)$ that depended on both the magnitude of the random variation and the interspecific variation in the measured traits:

$$
\operatorname{Dir}_{\text {res }}=\frac{\left(\sigma_{y \cdot p} / \sigma_{x \cdot p}\right)^{2}}{\left(\mathrm{SD}_{y} / \mathrm{SD}_{x}\right)},
$$

where $\sigma_{x \cdot p}$ and $\sigma_{y \cdot p}$ are the standard errors for values at a single point (taxon) and $\mathrm{SD}_{x}$ and $\mathrm{SD}_{y}$ are the standard deviations among the taxa (i.e., among the $x, y$ points for the regression). We designate this method least squares variance-oriented residuals (LSVOR) regression (see the appendix in the online edition of the American Naturalist for rationale and formulas).

In a preliminary assessment of the three regression methods, we simulated separate nonphylogenetic linear relationships with known levels of random noise in both the $x$ and $y$ variables to delineate under which conditions OLS, RMA, and LSVOR regressions would systematically underestimate or overestimate slopes. We started with values of the $x$ variable that were uniformly and randomly distributed between 0 and $x_{\max }$, with $x_{\max }$ in different simulations ranging from 1 to 10 . Linear relations were estimated as

$$
y=\beta \times x,
$$

where $\beta$ was a known slope with values ranging from 0.125 to 8 . Random variables for regression were then synthesized as

$$
\begin{aligned}
& x^{\prime}=x+\varepsilon_{x}, \\
& y^{\prime}=y+\varepsilon_{y},
\end{aligned}
$$

where $\varepsilon_{x}$ and $\varepsilon_{y}$ were normally distributed random errors with known standard deviations ranging from 0.01 to 10 . All results presented are for $x_{\max }=10$ (see the appendix).

\section{Phylogenetic Contrasts and Regressions}

Felsenstein's (1985) classic article pointed out the problems of (1) nonindependence of $x, y$ data pairs; (2) overestimation of available degrees of freedom for significance testing; and (3) the possibility of creating apparent regression relationships where none should exist when data are strongly phylogenetically structured. He also presented a partial solution that uses contrasts $(\Delta x, \Delta y)$ between sister taxa to make the $x, y$ pairs in the regression statistically independent and minimize bias due to shared evolutionary history. To prevent decimation of sample sizes, to allow comparison of distantly related taxa, and to allow comparison of "long branch" taxa (e.g., taxa at taxonomic orders 28,29 , and 50 in fig. 2), the method requires the creation of hypothetical ancestor species, with characters estimated from each of the descendant taxa using

$$
x_{\mathrm{anc}}=\frac{\left(1 / v_{\mathrm{a}-\mathrm{d} 1}\right) x_{\mathrm{d} 1}+\left(1 / v_{\mathrm{a}-\mathrm{d} 2}\right) x_{\mathrm{d} 2}}{\left(1 / v_{\mathrm{a}-\mathrm{d} 1}\right)+\left(1 / v_{\mathrm{a}-\mathrm{d} 2}\right)},
$$

where $x_{\text {anc }}$ is the estimated character value for the ancestor, $x_{\mathrm{d} 1}$ and $x_{\mathrm{d} 2}$ are the character values for the descendant, and $v_{\mathrm{a}-\mathrm{d} n}$ is the expected variance due to divergence between the ancestor and descendant number $n$. If estimated times of divergences (or some multiple thereof) are used as measures of the expected divergence between taxa, this is equivalent to

$$
x_{\mathrm{anc}}=\frac{\Delta t_{\mathrm{a}-\mathrm{d} 2} \times x_{\mathrm{d} 1}+\Delta t_{\mathrm{a}-\mathrm{d} 1} \times x_{\mathrm{d} 2}}{\Delta t_{\mathrm{a}-\mathrm{d} 2}+\Delta t_{\mathrm{a}-\mathrm{d} 1}},
$$

where $\Delta t_{\mathrm{a}-\mathrm{d} n}$ is the estimated interval between appearances of the ancestor and descendant number $n$. Further, one must increase the estimated times between taxa as specified by Felsenstein (1985). Garland et al. (1992) suggested that the contrasts should be standardized by dividing by a measure of the times or expected divergences between the taxa to prevent contrasts between more distantly related taxa from having undue leverage. Martins and Garland (1991) used simulated Brownian diffusive evolution to evaluate the use of Felsenstein's contrasts in phylogenetic regressions and found that slopes estimated using contrasts were less likely to be biased and had smaller variance than slopes estimated from uncontrasted (native) characters. 
We estimated contrasts four ways. First, we took character states directly from the recorded diffusion tree (tree method). The diffusion algorithm recorded the time and character states at each bifurcation, so ancestral states and divergence times were available without estimation. Second, we started with the terminal taxa and estimated ancestral character states via equations (7) and (8) and adjusted divergence times as suggested by Felsenstein (1985; Fels method). In this simplest approach, we specified contrasts simply as the difference between the character values for the two taxa. In addition, we scaled each of the above contrasts by dividing by the total divergence time (taxon 1 back to nearest common ancestor and then down to taxon 2, scaled tree and scaled Fels methods) as suggested by Garland et al. (1992) and commonly done for phylogenetically independent contrast analyses (Rohlf 2001). In addition, we calculated regressions on the uncontrasted character states (native data method).

\section{Computations}

All calculations were performed in MATLAB 7.0 (release 14; MathWorks, Natick, MA, http://www.mathworks .com). The RMA regressions were calculated from the eigenvectors of the covariance matrix of the standardized variables (correlation matrix). Random numbers, both normally distributed for diffusion steps and uniformly distributed for bifurcation probabilities, were those provided by MATLAB's standard random number generators (RANDN and RAND, respectively). MATLAB routines are available from the authors upon request.

\section{Results}

Model 1 versus Model 2 Regression: Synthetic Data without Phylogenetic Structure

Exploratory simulations with known random errors in both synthetic $x$ and $y$ variables suggested that OLS, RMA, and LSVOR regressions each performed well under certain conditions (fig. Al in the online edition of the American Naturalist). We focus on the systematic errors in the slope estimates. The MA regression rarely outperformed other methods in our simulations and is not presented. All results presented are for $x_{\max }=10$ and for $100 x, y$ pairs. Two thousand five hundred data sets were simulated for each combination of random error magnitudes. The results presented are typical of those for other known slopes (fig. A1). Both OLS and RMA regression worked well under the conditions for which they were most appropriate (OLS when $\sigma_{y \cdot p} / \sigma_{x \cdot p}$ was large and RMA when $\left.\sigma_{y \cdot p} / \sigma_{x \cdot p}=1\right)$. Under most other conditions, the LSVOR method outperformed both OLS and RMA. Even LSVOR regression is biased when $\sigma_{y \cdot p} / \sigma_{x \cdot p}$ is small $(<0.1)$. In this case, one would reverse the definitions of which variable was $x$ and which was $y$ and then repeat the analysis.

\section{Evolution with Rapid Early Diversification}

We simulated trees with rapid early diversification by decreasing $\sigma_{T}$ 10-fold when the number of extant taxa reached the square root of the target (i.e., about halfway through the simulation). This significantly increased the phylogenetic signal in the simulated data $\left(\mathrm{SS}_{\mathrm{b} / \mathrm{w}} / \mathrm{SS}_{\mathrm{w} / \mathrm{in}}=\right.$ 4-70 compared with $\sim 1.3$ with constant $\sigma_{T}$ ). We first asked whether different methods of estimating slopes (both with and without phylogenetic contrasts) affected slope estimates. We simulated 1,000 phylogenies, each with 100 terminal taxa. We estimated slopes of relationships between two of the simulated characters (expected slope $=0.75$ ), both from the native uncontrasted data and with the four methods of constructing phylogenetic contrasts listed above.

Regression slopes using Felsenstein's ancestor reconstruction (Fels method) correlated most closely with those using the tree method contrasts $\left(r^{2}=0.65\right.$, fig. 3$)$. Scaling either contrast type for expected divergence decreased the correlation $\left(r^{2}=0.2\right)$. Scaling the contrasts also slightly increased the variance of the estimated slopes in the 1,000 trees $\left(F\right.$ ratio $=1.53-1.86, P<10^{-6}$ via a resampling test for each error multiplier). Using uncontrasted (native) data led to low correlation with the contrasted slopes $\left(r^{2}=0.05-0.15\right.$; fig. 3$)$ and significantly increased variance in slope estimates ( $F$ ratio $=8-10, P<10^{-6}$ for each error multiplier). Significance of $F$ ratios was tested via a resampling test because of the correlation between the slopes for each simulated tree.

Different methods of constructing contrasts affected the estimated strength of the correlation among characters (fig. 4). Uncontrasted (native) data gave higher $r^{2}$ values than did the tree or Felsenstein contrasts, presumably because of the artifactual inclusion of phylogenetic signal in the character correlation (fig. 4). Additionally, scaling contrasts by expected diversification gave lower $r^{2}$ values than did the unscaled contrasts. The baseline value of $\sigma_{T}$ (initial rate of diversification) did not affect the strength of the correlation between character values (data not shown).

The OLS underestimated and RMA overestimated the expected parametric slopes ( 0.75 for these characters) when the ratio of random variance components $\left(E_{y} / E_{x}\right)$ was not optimal; LSVOR, however, provided better estimates of the parametric slope under most circumstances (fig. 5). The use of native uncontrasted data led to increased variance in slope estimates. For equivalent random error variances $\left(\sigma_{x}\right.$ in eq. [2]), using native data resulted in variances in slopes about 2 to 3 times those from using 

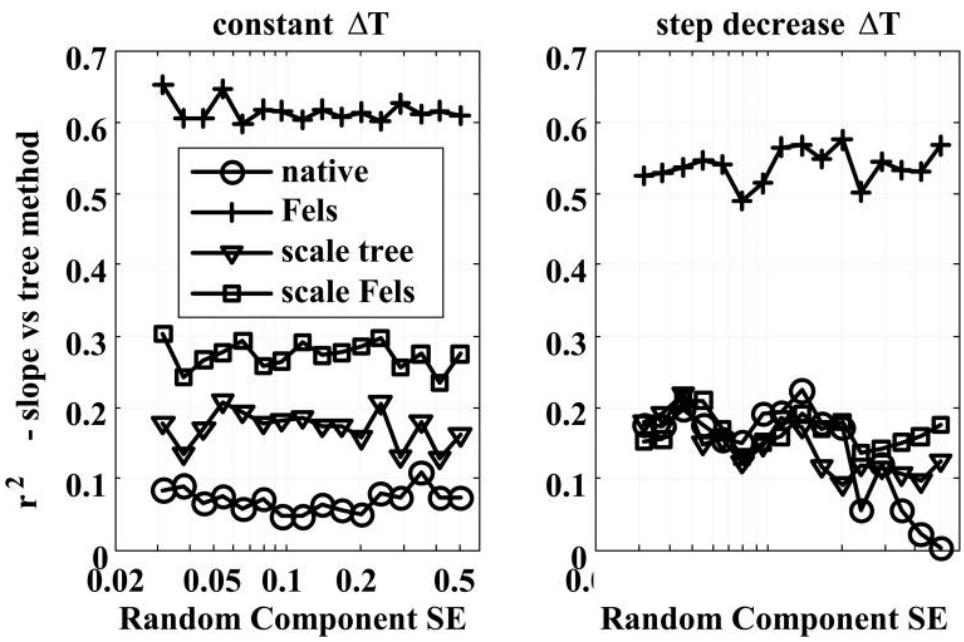

Figure 3: Correlation between least squares variance-oriented residuals slopes estimated on 1,000 replicate trees using native uncontrasted character values and four methods for constructing phylogenetic contrasts. Vertical axis gives $r^{2}$ of slopes via each method to those via tree contrast method versus size of random component in character diffusion. Left, simulations with constant rate of evolution over time. Right, simulations with step decrease in evolution rate. All results shown have same random variation for both $x$ and $y$ characters.

tree contrasts $\left(P<10^{-5}\right.$ by resampling test). Because estimated $r^{2}$ values differed among methods of constructing contrasts (fig. 4) and the variance of the slope estimate decreased as $r^{2}$ increased, variances in slope estimates for native data were eight to 10 times those for contrasts at equivalent $r^{2}$ values (SD ratio $=3$ ). Variances of slopes estimated using scaled contrasts were 1.15 to 1.30 times those for the corresponding unscaled contrast $(P=$ $.001-.1$ via resampling test). For $r^{2}$ values below 0.15, RMA and, to a lesser extent, LSVOR gave slope estimates that were highly variable and tended to misestimate the expected slope (data not shown). One of the limitations of RMA regressions is that for weak correlations, the estimated slope, instead of converging on 0 as occurs with OLS regression, becomes sensitive to the random variation in the data. Estimated slopes fell into two disjunct populations, one near the expected value of 0.75 and another near -0.75 . In our simulations, this sensitivity to noise occurred for $r^{2}$ values below 0.15 .

\section{Evolution at Constant Rates}

When $\sigma_{T}$ was kept constant throughout the simulation (constant evolutionary rate), phylogenetic signal as measured by the ratio of between-clade to within-clade variances was smaller than when $\sigma_{T}$ decreased during the simulation (rapid early divergence) but was still significant in most replicate simulations. Unlike simulations with rapid early diversification (fig. 4), use of phylogenetic contrasts did not strongly affect the strength of the correlation be- tween characters in the constant evolution simulations (data not shown).

Both the regression model employed and the use of phylogenetic contrasts affected estimated slopes. As with the rapid early diversification simulations (fig. 5), OLS regression tended to underestimate the parametric slope unless the $x$ character showed little variation $\left(E_{y} \gg E_{x}\right)$.

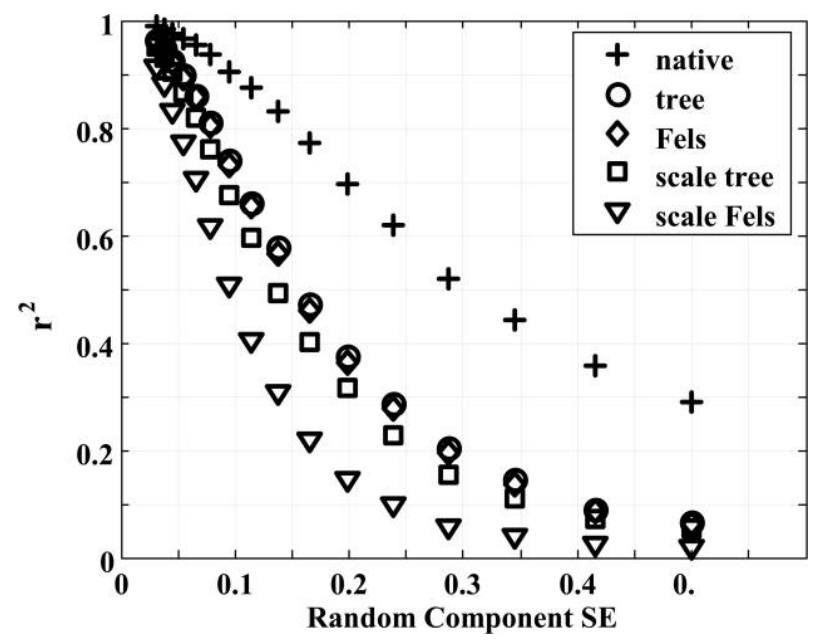

Figure 4: Estimated $r^{2}$ between simulated characters using both native uncontrasted character values and phylogenetic contrasts for regressions in simulations of evolution with rapid early divergence. Each value is the mean for 1,000 replicate trees with 100 terminal taxa. $\sigma_{T}=0.1$. Using $\sigma_{T}=0.3$ or 1.0 gave virtually identical plots. 


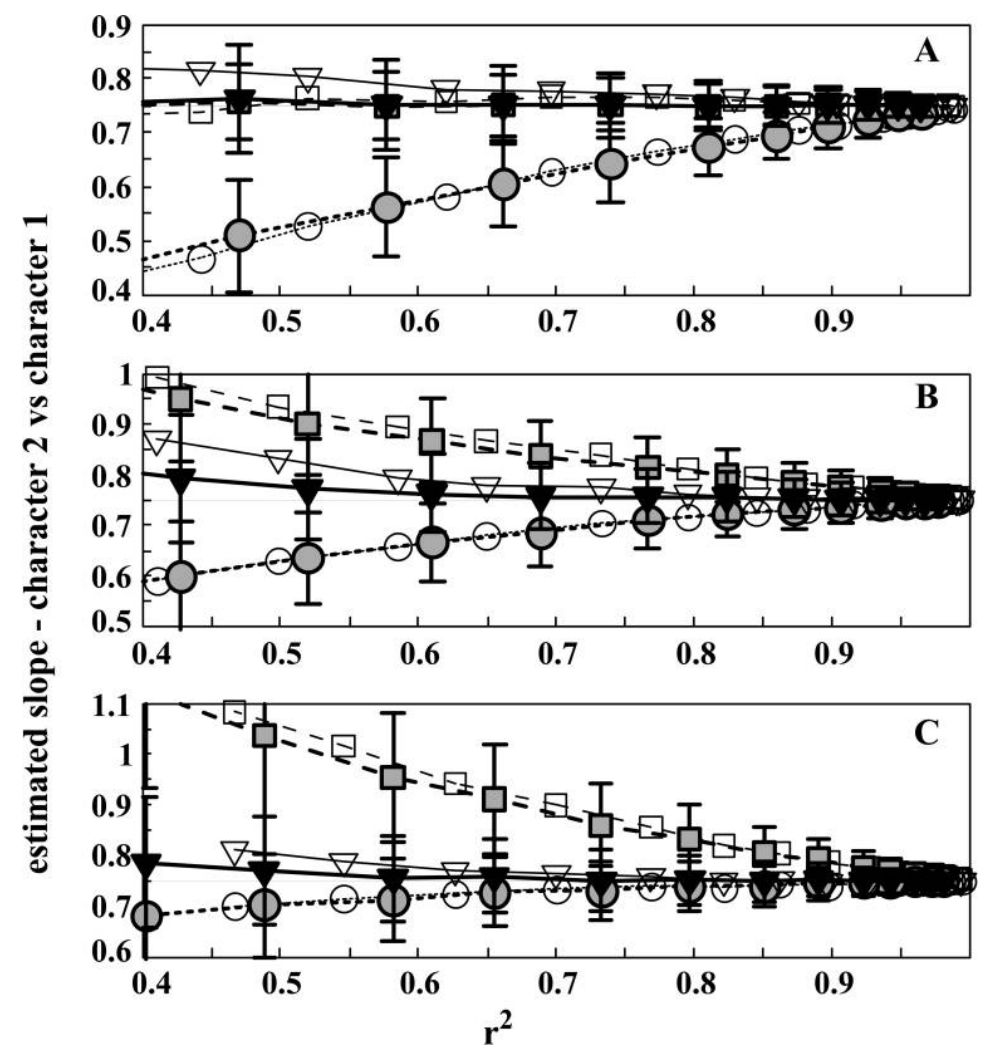

Figure 5: Slope estimates for both native uncontrasted character values and known tree contrasts for ordinary least squares (circles), reduced major axis (squares), and least squares variance-oriented residuals (triangles) regressions in simulations of evolution with rapid early divergence. Expected value of slope $=0.75$. Each value is the mean $( \pm 1 \mathrm{SD})$ for 1,000 replicate trees with 100 terminal taxa. Each panel presents results for a fixed ratio of error multipliers ( $E$ in eq. [2]) for the $x$ and $y$ characters. (top, $-E_{y} / E_{x}=1$; middle, $E_{y} / E_{x}=2 ;$ bottom, $\left.E_{y} / E_{x}=4\right)$ All values of $\sigma_{T}$ gave similar results, as did different methods of computing contrasts. For each regression method, heavier lines and filled symbols show slopes for regressions using known tree contrasts. Thinner lines with unfilled symbols give slopes for uncontrasted (native) data. Error bars for uncontrasted data were approximately twice those using contrasts but are not shown to enhance clarity.

Similarly, RMA regression slopes overestimated the parametric slope unless $E_{y}=E_{x}$ (data not shown). LSVOR regression performed well under most conditions despite variation in $E_{y} / E_{x}$. Using native (uncontrasted) data as input to any of the regressions did not bias the slope estimates but significantly increased the variance among slope estimates. For RMA regression, the $F$ ratio of variance in slopes estimates (from native data/from contrasts) ranged from eight to 10.5 ( $P<10^{-6}$ via resampling test).

\section{Extinctions}

We simulated the effects of extinction of taxa by censoring the simulated trees for both constant and variable $\sigma_{T}$. We present results only for slopes estimated using tree contrasts. We focus on simulations with a step decrease in evolutionary rate during the simulation (rapid early divergence). We present simulations for which $E_{y} / E_{x}=2$, which is not optimal for either OLS or RMA. We treated the individual tree as the unit of replication. For each tree, we averaged all slope estimates calculated with particular ranges of remaining taxa (e.g., 30-60 terminal taxa remaining). Figure 6 shows the means of those averages across the 100 original trees.

As the trees were progressively pruned, LSVOR slopes remained unbiased, approximating the expected slope of 0.75 . As the number of terminal taxa decreased toward $10 \%$ of the original number, there was some increase in the standard error of the slope estimate consistent with the smaller number of points in each regression (fig. 6). The OLS and RMA slopes became less strongly biased but not unbiased as the trees were pruned, and they also experienced some increase in the standard error of the slope estimate with progressive pruning (fig. 6). For each full tree (300 taxa), regression of the estimated slopes on the number of remaining taxa confirmed the patterns in figure 


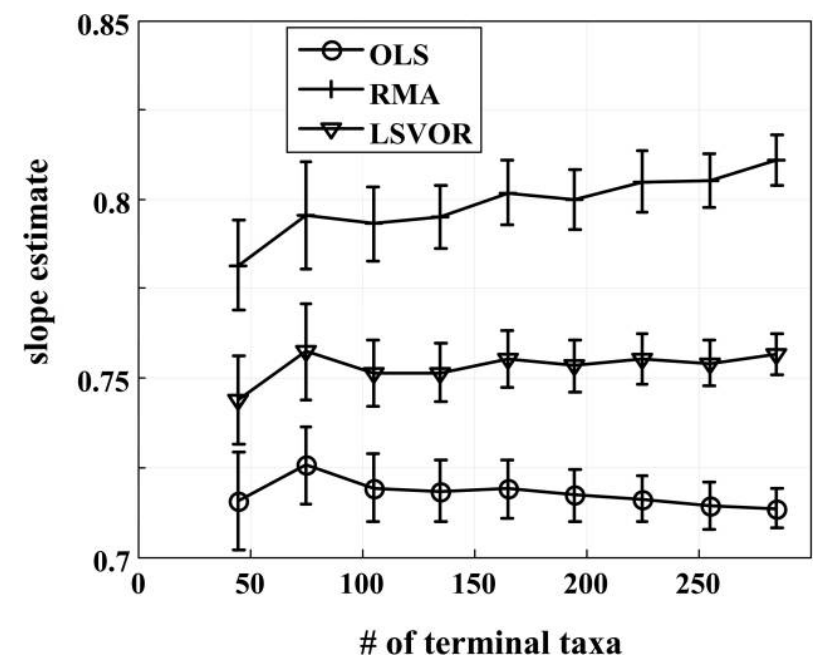

Figure 6: Slope estimates for RMA regressions of known tree contrasts for 100 replicate trees subjected to progressive random-node pruning to simulate the effect of extinction. Slopes were estimated by ordinary least squares (OLS), reduced major axis (RMA), and least squares varianceoriented residuals (LSVOR) regression. Each point is the among-trees mean of average of all estimated slopes for a particular tree in a range of remaining terminal taxa (bounds from 30 to 300 remaining taxa by steps of 30 ). Initial trees had approximately 300 terminal taxa. Error bars represent $95 \%$ confidence intervals on the mean.

6. For trees simulated using constant evolutionary rates, the increased dispersion of slope estimates as the trees were pruned persisted, but the tendency for OLS and RMA regressions to become less biased as the trees were pruned was abolished (data not shown). A few (1\%-2\%) trees as they were pruned to less than $30 \%$ of their original taxa yielded highly divergent slopes (e.g., 0 or negative slopes with parametric value 0.75 ) via both RMA and OLS techniques. This typically occurred after a step that pruned an early ancestral taxon and thus removed numerous taxa from the tree (data not shown).

\section{LSVOR and Residuals}

An alternate use of physiological phylogenetic regression is to use regression residuals to attempt to remove the portion of a signal that is correlated with another variable (e.g., Huey and Bennett 1987). The residuals considered are typically the deviations minimized by OLS regression, that is, the vertical deviation of the $y$ (dependent) value from the regression line. Using LSVOR complicates this process in two ways. First, the residuals usually computed are not those minimized by LSVOR, and vertical deviations from the LSVOR line will tend to be larger than the residuals from an OLS regression. Second, the residual minimized by LSVOR has both $x$ and $y$ components and is conceptually more complicated than the OLS residual (e.g., if the residual has both $x$ and $y$ components, what signal does using the LSVOR residual remove?). We examined vertical residuals (the distance from the data point to the regression line in the $y$ direction) for OLS, RMA, and LSVOR regressions and the LSVOR residual distance for 10,000 replicate trees with 100 terminal taxa for $E_{y} / E_{x}$ ratios ranging from one to eight. Three patterns emerge: (1) all four residual measurements are highly correlated (minimum $r^{2} \sim 0.8$ ); (2) for conditions under which OLS regression lines diverge most strongly from LSVOR $\left(E_{y} / E_{x}=1\right)$, vertical residuals from LSVOR and RMA lines average approximately $20 \%$ larger than OLS residuals, but the actual LSVOR residual distance is approximately $20 \%$ shorter than the OLS residual; and (3) when $E_{y} / E_{x}=8$, vertical residuals from OLS and LSVOR regressions differ by $<1 \%$, and the actual LSVOR residual distance is slightly shorter.

\section{Discussion}

Our simulations suggest that both the choice of regression model and the use of phylogenetic contrasts can affect estimated allometric exponents, though in different ways. Here, we address each of the three major questions that motivated the simulations, presenting both the effects seen in the simulations and the caveats in interpreting those effects.

\section{Regression Models}

The OLS (model 1) regressions significantly underestimated the expected value of the slope of the relationship among the characters resulting from our Brownian diffusive evolution simulation unless $E_{y} \gg E_{x}$ (fig. 5). Similarly, RMA (model 2) regressions significantly overestimated slopes unless $E_{y}=E_{x}$ (fig. 5). Alternatively, LSVOR regressions showed no significant bias for $r^{2}$ values $>0.2$. The simulations suggest that LSVOR regressions should be used under most conditions for physiological allometries with strong relationships between the variables.

Three considerations are vital in interpreting these results. First, in our simulations, both variables change in response to a third hidden variable, but each is subject to variation unrelated to the physiological constraints that generate the relationship. The simulations thus create characters, each of which has a random component and none of which are causal independent variables. The RMA (model 2) regression is usually advocated for such data (Sokal and Rohlf 1995; Quinn and Keough 2002; Warton et al. 2006), but RMA assumes that the random components for both $x$ and $y$ variables are similar (Carroll and Ruppert 1996). In our simulations, RMA performed well 
under conditions for which it was derived $\left(\sigma_{y \cdot p} / \sigma_{x \cdot p}=\right.$ $E_{y} / E_{x} \times 1$ ) but poorly under other conditions (figs. 3,5 ). The simplest summary of our results is that LSVOR outperformed OLS and RMA under some circumstances and performed as well as the other methods when they did well (figs. 3, 5). In fact, the virtue of LSVOR is that it behaves like OLS when $\sigma_{y \cdot p} / \sigma_{x \cdot p}=E_{y} / E_{x} \gg 1$, behaves like RMA when $\sigma_{y \cdot p} / \sigma_{x \cdot p}=E_{y} / E_{x}=1$, and grades smoothly between the two methods for intermediate data (see the appendix).

Second, there is no experimental measurement error in our simulations. The random variation in our simulated characters arises from noise in the Brownian diffusion process, not from errors in the measurement of the characters. Both sorts of random variation, biological and measurement, can contribute to important variation in characters. Thus, OLS is sometimes used for metabolic allometries because mass can often be measured more precisely than metabolic rate (e.g., Glazier 2005). But intraspecific variation in body mass, although perhaps precisely measured, creates a statistical "measurement error" related to sampling rather than to techniques of measurement. Such intraspecific variation in physiological characters will be virtually omnipresent, regardless of the magnitude of technical measurement error. Highly specialized regression models can be used depending on the magnitude, origin, and assumed statistical characteristics of random variation in characters (Draper and Smith 1998; Warton et al. 2006), but we argue that the magnitude of experimental measurement errors should not be the sole determinant of which regression model is used to analyze physiological allometric data.

Third, allometric regressions differ from some other physiological regressions in that intraspecific variation (both biological and measurement) provides measures of $\sigma_{x \cdot p}$ and $\sigma_{y \cdot p}$. The LSVOR regression requires such estimates to select the slope along which residuals are measured (eq. [4]; see the appendix). Without estimates of $\sigma_{x \cdot p}$ and $\sigma_{y \cdot p}$, the direction of the residual measurements must be made independently of the data, as in OLS and RMA regressions, and the LSVOR technique loses its flexibility and, hence, the advantages portrayed in figures 3 and 5 .

Taxon extinction partially ameliorated the systematic errors in estimated slope (OLS and RMA, rapid early divergence only), but significant estimation errors remain, and the reduced bias comes at the cost of increased variance in the estimated slopes (fig. 6). The absence of this pattern in simulations with constant evolutionary rates suggests that the "improvement" was due to extinction censoring the stronger phylogenetic signal in the rapid early divergence simulations. Thus, our simulations revealed little effect of extinction on the utility of different regression methods.

\section{Contrasts}

In our simulations, as in previous reports (Martins and Garland 1991), failure to use some type of phylogenetic contrasts overestimated the strength of the relationships between characters for strongly phylogenetically structured data (fig. 4) and increased variances of the estimated slopes 8-10-fold ( $\sim$ threefold increase in standard error of the estimate; fig. 5). Methods of estimating contrasts affect correspondence to the known contrasts recorded during the simulation (fig. 3), but effects on $r^{2}$ and estimated slopes (figs. 4,5 ) were more subtle and generally consisted of slight increases in the variance of the slope estimates. Slope estimates suggest no interaction between regression methodology and the need for contrasts (fig. 5). Our results neither strongly support nor dispute the scaling of contrasts by expected character divergence as recommended by Garland et al. (1992).

Thus, our results support the use of phylogenetic contrasts in constructing physiological allometries. One of the assumptions of such analyses, as identified by Felsenstein (1985; Garland et al. 1992) is that the structure of the phylogenetic tree used to construct the contrasts is correctly specified. Our results do not address to what extent regression results might be sensitive to uncertainties in either the connections or the branch lengths of the phylogenetic tree used in the analysis (Martins and Garland 1991; Garland et al. 1992). This is particularly important in physiological allometries because the taxonomic range of organisms considered is often extensive (e.g., Brown et al. 2004; West and Brown 2005), and well-resolved trees that span such taxonomic breadths (or methods to articulate more narrowly focused trees to those with broader taxonomic foci) are often lacking.

We concentrate here on relatively simple phylogenetic adjustment methods (Felsenstein's [1985] contrasts and scaled contrasts). A series of more recent and, in many cases, more flexible alternatives are available. Phylogenetic generalized linear models (Grafen 1989; Martins and Hansen 1997) allow for more general variance structures for contrasts as can be important when stabilizing selection or punctuated evolution are important characteristics of the phylogeny (Diaz-Uriarte and Garland 1996; Hansen and Martins 1996; Hansen 1997; Butler and King 2004) Phylogenetic mixed models (Housworth et al. 2004) and autocorrelation models (Cheverud and Dow 1985; Martins 1996) both try to partition variation into that associated with phylogeny and that associated with other factors. We concentrate on simple contrasts in preference to those models here for several reasons. First, phylogentically independent contrasts are the most simple, basic, and primitive of the approaches. Second, we used a modified, constrained Brownian diffusion model, and phylogentically 
independent contrasts account for the effects of phylogeny as well as other approaches under those circumstances (Rohlf 2001). Third, some of the methods (e.g., autocorrelation methods; Martins 1996) assume that variation within taxa is negligible, whereas we sought a solution for problems where the variation around individual points was present and measurable. Fourth, and perhaps most importantly, many more advanced methods require a distinction between regressors and response variables in the analysis (Rohlf 2001). This asymmetry between causal and response variables is the model for OLS regression. The RMA and LSVOR regressions are symmetric with neither variable necessarily designated as causal or error free. Thus, we chose to use the more basic phylogenetically independent contrasts, which can be easily adapted to OLS, RMA, and LSVOR regression. How LSVOR regressions might be incorporated into phylogentic generalized least squares or other models is an interesting question for further study.

\section{Allometries}

Functional allometries differ from some other regression problems not only in the importance and estimableness of their variance structure but also in the objectives of the analysis. The biological and conceptual importance of slopes estimated by regression, and disagreement over those slopes (Brown et al. 2004; West and Brown 2005; White and Seymour 2005) make estimation of slopes rather than estimation of particular characters, the major objective of allometric analyses in many cases. With this "error-in-variables" variance structure and focus on the regression slope, the consensus advice on regression techniques is that some sort of model 2 regression should be used (Sokal and Rohlf 1995; Quinn and Keough 2002; see recent review in Warton et al. 2006) despite a dearth of software that allows the simultaneous use of phylogenetically corrected and model 2 regression techniques (Martin et al. 2005; Warton et al. 2006). Our simulation results however, argue that both OLS and RMA regression systematically misestimate allometric regression slopes but that LSVOR, a variant of orthogonal regression (Fuller 1987), approximates RMA and OLS under some circumstances and provides less biased estimates of regression slopes under others (fig. 5). Our simulations further support previous work suggesting that failure to use one $o \rightarrow$ several sets of phylogenetic contrasts results in less precise slope estimates (fig. 5) and can overestimate $r^{2}$ (fig. 4) and, thus, inflate our confidence in the estimated slope.

Using LSVOR regression on physiological phylogenetir contrasts is more technically demanding than current practices because it requires (1) a phylogeny for the specie: of interest, which may require concatenation of component phylogenetic trees; (2) an estimate of $\sigma_{y \cdot p} / \sigma_{x \cdot p}$, which our method assumes is constant for all species in the analysis and which will need to be extracted from original reports; and (3) computational techniques that are not already assembled in most statistical packages. However, contrasts can be constructed by numerous software packages, and the LSVOR regression is relatively simple to program (MATLAB routine available from the authors).

When the phylogeny and variance estimates are available, our simulations suggest that less biased and less variable estimates of the regression slopes result from using LSVOR as opposed to OLS or RMA techniques. Thus, applying LSVOR techniques to allometric and other phylogenetic regressions where the slope of the relationship is critical would be an important first step to discussing the import of the estimated slopes.

\section{Acknowledgments}

We thank B. Wallace for helpful discussions in formulating our simulations. We also thank two anonymous reviewers for useful comments on an earlier version of the article. F.H. was partially supported by a cooperative agreement from the U.S. Department of Agriculture's National Wildlife Research Center (03-7408-0479-CA) to the University of Pennsylvania.

\section{Literature Cited}

Brown, J. H., J. F. Gillooly, A. P. Allen, V. M. Savage, and G. B. West. 2004. Toward a metabolic theory of ecology. Ecology 85:17711789.

Butler, M. A., and A. A. King. 2004. Phylogenetic comparative analysis: a modeling approach for adaptive evolution. American Naturalist 164:683-695.

Calder, W. A., III. 1984. Size, function and life history. Harvard University Press, Cambridge, MA.

Carroll, R. J., and D. Ruppert. 1996. The use and misuse of orthogonal regression in linear errors-in-variables models. American Statistician 50:1-6.

Cheverud, J. M., and M. M. Dow. 1985. An autocorrelation analysis of genetic variation due to lineal fission in social groups of rhesus macaques. American Journal of Physical Anthropology 67:113121.

Diaz-Uriarte, R., and T. Garland Jr. 1996. Testing hypotheses of correlated evolution using phylogenetically independent contrasts: sensitivity to deviations from Brownian motion. Systematic Biology 45:27-47.

Dodds, P. S., D. H. Rothman, and J. S. Weitz. 2001. Re-examination of the "3/4-law" of metabolism. Journal of Theoretical Biology 209:9-27.

Draper, N. R., and H. Smith. 1998. Applied regression analysis. 3rd ed. Wiley, New York.

Felsenstein, J. 1985. Phylogenies and the comparative method. American Naturalist 125:1-15.

Freckleton, R. P., P. H. Harvey, and M. Pagel. 2002. Phylogenetic analysis and comparative data: a test and review of evidence. American Naturalist 160:712-726. 
Fuller, W. A. 1987. Measurement error models. Wiley, New York.

$\rightarrow$ Garland, T., P. H. Harvey, and A. R. Ives. 1992. Procedures for the analysis of comparative data using independent contrasts. System atic Biology 41:18-32.

$\rightarrow$ Gillooly, J. F., A. P. Allen, G. B. West, and J. H. Brown. 2005. The rate of DNA evolution: effects of body size and temperature or the molecular clock. Proceedings of the National Academy of Sciences of the USA 102:140-145.

$\rightarrow$ Glazier, D. S. 2005. Beyond the "3/4-power law": variation in the intra- and interspecific scaling of metabolic rate in animals. Biological Reviews 80:611-662.

$\rightarrow$ Grafen, A. 1989. The phylogenetic regression. Philosophical Transactions of the Royal Society B: Biological Sciences 326:119-157.

$\rightarrow$ Hansen, T. F. 1997. Stabilizing selection and the comparative analysis of adaptation. Evolution 51:1341-1351.

$\rightarrow$ Hansen, T. F., and E. P. Martins. 1996. Translating between microevolutionary process and macroevolutionary patterns: the correlation structure of interspecific data. Evolution 50:1404-1417.

$\rightarrow$ Housworth, E. A., E. P. Martins, and M. Lynch. 2004. The phylogenetic mixed model. American Naturalist 163:84-96.

Huey, R. B., and A. F. Bennett. 1987. Phylogenetic studies of coadaptation: preferred temperatures versus optimal performance temperatures of lizards Evolution 41:1098-1115.

$\rightarrow$ Isler, K., A. D. Barbour, and R. D. Martin. 2002. Line-fitting b; $\rightarrow$ rotation: a nonparametric method for bivariate allometric analysis. Biometrical Journal 44:289-304.

$\rightarrow$ Jolicouer, P. 1975. Linear regression in fishery research: some comments. Journal of the Fisheries Research Board of Canada 32:14911494.

$\rightarrow$ Jolicouer, P., and A. A. Heusner. 1971. The allometry equation in the analysis of the standard oxygen consumption and body weight of the white rat. Biometrics 27:841-855.

$\rightarrow$ LaBarbera, M. 1989. Analyzing body size as a factor in ecology and evolution. Annual Review of Ecology and Systematics 20:97-117.

Legendre, P., and L. Legendre. 1998. Numerical ecology. 2nd English ed. Elsevier Science, Amsterdam.

$\rightarrow$ Martin, R. D., M. Genoud, and C. K. Hemelrijk. 2005. Problems of allometric scaling analysis: examples from mammalian reproductive biology. Journal of Experimental Biology 208:1731-1747.

$\rightarrow$ Martins, E. P. 1996. Phylogenies, spatial autoregression, and the com- parative method: a computer simulation test. Evolution 50:17501765.

Martins, E. P., and T. Garland. 1991. Phylogenetic analysis of the correlated evolution of continuous characters: a simulation study. Evolution 45:534-557.

Martins, E. P., and T. F. Hansen. 1997. Phylogenies and the comparative method: a general approach to incorporating phylogenetic information into the analysis of interspecific data. American Naturalist 149:646-667.

Peters, R. H. 1983. The ecological implications of body size. Cambridge University Press, Cambridge.

Quinn, G. P., and M. J. Keough. 2002. Experimental design and data analysis for biologists. Cambridge University Press, Cambridge.

$\rightarrow$ Rohlf, F. J. 2001. Comparative methods for the analysis of continuous variables: geometric interpretations. Evolution 55:2143-2160.

$\rightarrow$ Savage, V. M., J. F. Gillooly, W. H. Woodruff, G. B. West, A. P. Allen, B. J. Enquist, and J. H. Brown. 2004. The predominance of quarterpower scaling in biology. Functional Ecology 18:257-282.

Schmidt-Nielsen, K. 1984. Scaling: why is animal size so important? Cambridge University Press, Cambridge.

Sokal, R. R., and F. J. Rohlf. 1995. Biometry. 3rd ed. W. H. Freeman, New York.

Symonds, M. R., and M. A. Elgar. 2002. Phylogeny affects estimation of metabolic scaling in mammals. Evolution 56:2330-2333.

Warton, D. I., I. J. Wright, D. S. Falster, and M. Westoby. 2006. Bivariate line-fitting methods for allometry. Biological Reviews 81: 259-291.

$\rightarrow$ West, G. B., and J. H. Brown. 2005. The origin of allometric scaling laws in biology from genomes to ecosystems: towards a quantitative unifying theory of biological structure and organization. Journal of Experimental Biology 208:1575-1592.

West, G. B., J. H. Brown, and B. J. Enquist. 1997. A general model for the origin of allometric scaling laws in biology. Science 276: 122-126.

White, C. R., and R. S. Seymour. 2003. Mammalian basal metabolic rate is proportional to body mass ${ }^{2 / 3}$. Proceedings of the National Academy of Sciences of the USA 100:4046-4049.

$\rightarrow \frac{\text {. 2005. Allometric scaling of mammalian metabolism. Journal }}{\text {. }}$ of Experimental Biology 208:1611-1619.

Associate Editor: Emília P. Martins Editor: Michael C. Whitlock 